\title{
Development and process evaluation of a brief intervention on diet and CHD prevention in a university hospital primarycare outpatient clinic
}

\author{
M. Kruseman ${ }^{1}$, D. Lamalle ${ }^{1}$, M. Prod'hom ${ }^{1}$, A. Rieder Nakhlé ${ }^{2}$ and J. M. Gaspoz ${ }^{2}$ \\ ${ }^{1}$ University of Applied Sciences Western Switzerland, Dietetics and Nutrition, Geneva, Switzerland and ${ }^{2}$ Division of \\ Primary Care Medicine, Geneva University Hospitals, Geneva, Switzerland
}

Dietary counselling is an important aspect of CHD prevention. However, at-risk patients may be reluctant to visit a dietitian. General practitioners (GP) could participate in the CHD prevention effort, but often have difficulty when counselling patients on their diet. A team of dietitians and physicians have designed a five-step brief intervention (BI) to be used by GP. The aim was to evaluate the feasibility and acceptability of this dietary BI among physicians and patients.

The content of the BI was based on published recommendations on dietary CHD prevention and its design was supervised by a physician experienced in BI technique. Five GP in a primarycare setting agreed to test the BI among their patients for 1 month and participated in two $2 \mathrm{~h}$ training sessions. Two trainee dietitians approached the patients and asked them to fill in a screening questionnaire on their dietary habits. Answers were used by the physician to define, along with a decision algorithm, the content of a short counselling session that was integrated into the routine medical visit. The BI process and its acceptability by patients and physicians were assessed by means of standardised interviews and questionnaires.

Of seventy-six patients included, forty-nine (65\%) received the BI, which took 5-10 min in a 30 min consultation. Reasons for physicians not to implement the BI were 'lack of time' $(n$ 20) or 'no interest of the patient' ( $n$ 7). Three of the five steps were performed $>70 \%$ of the time. All physicians rated the tool as 'useful' and their average motivation score remained high during the study period: 6.8 (SD 1.1)-6.3 (SD 1.4) on a ten-point scale. Of the forty-nine patients who received the BI, thirty-six (78\%) said that they had learned something new about their diet in relation to CHD and thirty-one $(64 \%)$ were able to identify at least one dietary habit they planned to change. Of the patients $98 \%$ thought it was their physician's role to discuss their diet to prevent CHD.

This BI on diet and CHD prevention seemed to be functional and acceptable in general medical practice. Patients and physicians rated the tool as useful. Most patients identified dietary habits they could change. The next objective is to evaluate the ability of this BI to modify patients' dietary behaviour. 